\title{
Computer-aided diagnosis of school-aged children with ASD using full frequency bands and enhanced SAE: A multi-institution study
}

\author{
ZHIYONG XIAO ${ }^{1,2}$, JIANHUA WU ${ }^{3}$, CANHUA WANG $^{1,4}$, NAN JIA $^{3}$ and XIAOLING YANG ${ }^{5}$ \\ ${ }^{1}$ School of Mechatronics Engineering, Nanchang University, Nanchang, Jiangxi 330031; ${ }^{2}$ School of Software, \\ Jiangxi Agricultural University, Nanchang, Jiangxi 330045; ${ }^{3}$ School of Information Engineering, Nanchang University, \\ Nanchang, Jiangxi 330031; ${ }^{4}$ School of Computer Sciences, Jiangxi University of Traditional Chinese Medicine, Nanchang, \\ Jiangxi 300004; ${ }^{5}$ School of Engineering, Jiangxi Agricultural University, Nanchang, Jiangxi 330045, P.R. China
}

Received July 5, 2018; Accepted February 6, 2019

DOI: $10.3892 /$ etm.2019.7448

\begin{abstract}
Autism spectrum disorder (ASD) is a neurodevelopmental and network-level disorder mainly diagnosed in children. The aim of the current study was to develop a computer-aided diagnosis method with high accuracy to distinguish school-aged children (5-12 years) with ASD from those typically developing (TD). The current study used multi-institutional functional magnetic resonance imaging (fMRI) datasets of 198 school-aged participants from the Autism Brain Imaging Data Exchange II database and employed enhanced stacked auto-encoders to distinguish between school-aged children with ASD from those TD. In the current study, the average diagnostic accuracy was $96.26 \%$ (average sensitivity $=98.03 \%$; average specificity $=93.62 \%$ ); these results of classification were higher than that observed in previous studies using single or two frequency bands. The current study demonstrated that the proposed computer-aided diagnosis method may be used to distinguish between school-aged children with ASD from those TD. Attempts to use full frequency bands, deep learning based algorithm and multi-institutional fMRI datasets to distinguish between school-aged children with ASD from TD may be a key step towards clinical auxiliary diagnosis independent of sex, handedness, intellectual level or scanning parameters of fMRI data.
\end{abstract}

\section{Introduction}

Autism spectrum disorder (ASD) is a neurodevelopmental and network-level disorder characterized by impaired development of social interaction, communication skills and a restricted repertoire of activities and interests (1). In 2014,

Correspondence to: Dr Jianhua Wu, School of Information Engineering, Nanchang University, 999 Avenue of Xuefu, Nanchang, Jiangxi 330031, P.R. China

E-mail: jhwu@ncu.edu.cn

Key words: computer-aided diagnosis, school-aged children, autism spectrum disorder, stacked auto-encoders the Centers for Disease Control and Prevention (CDCP) estimated that 1 in 68 children were identified with ASD in the United States, an increase of $\sim 30 \%$ compared with previous estimates of 1 in 88 children identified with ASD in 2012 (2,3). In 2014, China's Health and Family Planning Commission reported that an estimated 41,000 children aged 0-6 years were identified with ASD (4). ASD mostly affects language, social communication, motor behaviors and sensory systems (5). Therefore, parents/guardians of children with ASD should consult with pediatric specialists as early as possible, to begin rehabilitation and avoid abuse of diagnosis (6). Currently, the diagnosis of children with ASD is based mostly on behavioral phenotypes (7), which include Autism Diagnostic Interview-Revised (ADI-R) (8), Autism Diagnostic Observation Schedule (ADOS) (9) and the Diagnostic and Statistical Manual of Mental Disorders, Fourth Edition-Text Revision (10). In the United States, only $8 \%$ of pediatric specialists capable of conducting ASD screening and diagnosis routinely evaluate toddlers for autism (11). In 2014, China's Health and Family Planning Commission reported that there were $\leq 100$ doctors nationwide trained in diagnosing autism (4). Additionally, the majority of ASD diagnostic tools are not available for use in school-age children. Thus, there is an urgent need for auxiliary diagnosis tools, which may aid to distinguish children with ASD from those typically developing (TD), in particular school-aged children.

Currently, there are several commonly used diagnostic methods and auxiliary diagnostic tools, which include diagnostic methods based on language, behavior, genes, animal models and/or functional magnetic resonance imaging (fMRI) data (12). With the development of interdisciplinary technology, a diagnostic method based on multi-modulus data and machine learning has become popular in recent years (13-16). One of the most effective methods is based on brain frequency and machine learning (14,15,17-19). Zuo et al (20) proposed a concept of brain frequency band and divided the brain frequency band into four frequency sub-bands Slow-2 (0.198-0.25 Hz), Slow-3 (0.073-0.198 Hz), Slow-4 (0.027-0.073 Hz) and Slow-5 (0.01-0.027 Hz). Other studies also demonstrated that the method of diagnosing autism based on brain frequency was possible (21-24). These studies also demonstrated that different brain frequency bands 
revealed different properties and physiological functions and they hypothesized that these could be the 'fingerprints' of neuronal activity (25). However, these investigations based on brain frequencies demonstrated limited use for the classification of children with ASD and those TD. In addition, the accuracy of classification decreased when the fMRI data was obtained from a single institution (19). Chen et al (19) used fMRI data from multiple institutions to distinguish ASD from TD and demonstrated a 74\% diagnostic accuracy when using two frequency bands compared with a $69 \%$ diagnostic accuracy when using only one frequency band. The heterogeneity and complexity of ASD has impaired the research of ASD in children (26). Therefore, methods based on one frequency band and traditional machine learning cannot be used for the auxiliary diagnosis of ASD. Full brain frequency bands contain more information, however they also have some noise, such as physiological noise (20). The stacked auto-encoders (SAE) deep learning framework is a neural network consisting of multiple layers and it can extract important features from input data (27). In the current study, an advanced deep learning method based on SAE, full frequency bands and multi-institutional fMRI data was used to distinguish school-aged children with ASD from those TD. The current study examined large fMRI datasets of 198 school-aged children from three different institutions obtained from the Autism Brain Imaging Data Exchange (ABIDE) II database (fcon_1000.projects.nitrc.org/indi/abide/index.html) (28) and demonstrated relatively high classification accuracy compared with previous studies $(16,19,29-31)$.

\section{Materials and methods}

Data collection. Original fMRI datasets of 198 school-aged children (5-12 years) were downloaded from the ABIDE II database, which was released in June 2016 and enables unrestricted use for non-commercial purposes. The current study was approved by the Ethics Committee of Nanchang University (Nanchang, China).

Patient charts. The current study used original fMRI datasets of school-aged children obtained from the New York University Langone Medical Center (New York, NY, USA), Georgetown University (Washington, DC, USA) and the Kennedy Krieger Institute (Baltimore, MD, USA). For each participant, a high-resolution structural MRI sequence was acquired using different MRI scanners with different parameters, as well as a resting-state fMRI sequence using different MRI scanners with different parameters. The names of the three different institutions and the different fMRI scanning parameters are summarized in Table I.

All participants were diagnosed according to the score obtained in both the ADI-R and the ADOS. The TD participants were screened during clinical interviews conducted by child psychiatrists and details of the diagnostic procedures and questionnaires are listed in the ABIDE II database (28). According to age, sex and full-scale intelligence quotient (FIQ), 117 participants with ASD (minimum age, 5.22 years; maximum age, 11.99 years; mean age [standard deviation (SD)], 9.32 [5.62] years; FIQ [SD], 107.87 [15.26]) and 81 ageand FIQ-matched TD participants [minimum age, 5.88 years; maximum age, 11.95 years; mean age (SD), 9.67 (5.31); mean FIQ (SD), 109.40 (15.12)] were selected from the datasets obtained from New York University Langone Medical Center, Georgetown University and the Kennedy Krieger Institute. Demographic data for the selected participants are summarized in Table II.

Resting-state fMRI data preprocessing. Resting-state fMRI preprocessing was performed using the multivariate exploratory linear optimized decomposition into independent components (MELODIC; version 3.14), a tool of FMRIB Software Library (FSL) software packages (32). Data preprocessing included head motion correction slice timing correction, removal of non-brain tissues, and spatial normalization into MNI152 space using nonlinear registration and spatial smoothing using $6 \mathrm{~mm}$ full width half maximum Gaussian kernel. Currently, the prediction of autism using brain networks involves examining functional connectivity in 10 functional networks using independent component analysis (33). A previous study identified 30 independent components (ICs) corresponding to 10 previously described functional networks (34). By contrast, several studies revealed that the number of ICs increases to 50, additionally the time of fMRI data decomposition increases exponentially and the dimension of obtaining brain frequency signals also increases $(26,35)$. High-dimensional brain signals will directly make the neural node of the deep learning algorithm increase, which will also increase the training time of the system. Therefore, each participant's data were decomposed into 30 ICs using MELODIC (36).

Frequency selection and normalization. Following IC decomposition using MELODIC version 3.14, the time serial, the power spectrum and the spatial map are generated for each IC (Figs. 1-3). MELODIC can generate the ICs on one spatial map followed by the relevant time serial of the IC and the power spectrum of the time serial. The power spectrum is an expression of brain frequency power distribution. The full brain frequency was divided into 5 different bands: Slow-6 (0-0.01 Hz), Slow-5 (0.01-0.027 Hz), Slow-4 (0.027-0.073 Hz), Slow-3 (0.073-0.198 Hz) and Slow-2 (>0.198 Hz). The power spectra of 30 ICs for each participant contain the information of each sub-frequency band in the network. In order to get more information, all ICs were selected in a data-driven manner although some ICs may contain useless information such as noise. All ICs with full brain frequency were selected according to the frequency ranges, although some ICs may contain low frequency drifting and noise.

For each participant, a time-series of all ICs were reshaped into a feature array with the same dimension. The feature array was normalized in the range of $(0,1)$ using Max-Min normalization before it was input into the SAE-based classifier.

SAE-based classification. In general, a sparse auto-encoder (AE) is used for feature extraction and dimensionality reduction. The sparse AE is a framework of unsupervised learning method, and SAE is a neural network consisting of multiple layers of sparse AE, where the output of each layer is connected to the input of each successive layer (35). A softmax classifier is used for phase classification, a supervised learning approach (37). SAE and softmax classifier 
Table I. fMRI scanning parameters and settings.

\begin{tabular}{|c|c|c|c|c|c|c|c|}
\hline Institution & $\begin{array}{c}\text { MRI } \\
\text { manufacturer }\end{array}$ & $\begin{array}{c}\mathrm{TR} \\
(\mathrm{msec})\end{array}$ & $\begin{array}{c}\mathrm{TE} \\
(\mathrm{msec})\end{array}$ & $\begin{array}{l}\text { Voxel } \\
\left(\mathrm{mm}^{3}\right)\end{array}$ & Slices & $\begin{array}{c}\text { Scan } \\
\text { time }(\min )\end{array}$ & Eyes \\
\hline Georgetown University & Siemens AG, Munich, Germany & 2,000 & 30 & $3.00 \times 3.00 \times 2.50$ & 43 & 5.23 & Open \\
\hline Kennedy Krieger Institute & $\begin{array}{l}\text { Philips Medical Systems, Inc., } \\
\text { Bothell, WA, USA }\end{array}$ & 2,500 & 30 & $2.67 \times 2.67 \times 3.00$ & 47 & 6.67 & Open \\
\hline $\begin{array}{l}\text { New York University } \\
\text { Langone Medical Center }\end{array}$ & Siemens AG, Munich, Germany & 2,000 & 30 & $3.00 \times 3.00 \times 3.00$ & 34 & 6.00 & Open \\
\hline
\end{tabular}

fMRI, functional magnetic resonance imaging; MRI, magnetic resonance imaging; TR, repetition time; TE, echo time.

Table II. Demographic data of ASD and TD participants.

\begin{tabular}{lccccc}
\hline $\begin{array}{l}\text { Participant } \\
\text { group }\end{array}$ & Patients (n) & $\begin{array}{c}\text { Male/female } \\
\text { ratio }\end{array}$ & $\begin{array}{c}\text { Minimum } \\
\text { age, years }\end{array}$ & $\begin{array}{c}\text { Maximum } \\
\text { age, years }\end{array}$ & $\begin{array}{c}\text { Mean age, } \\
\text { years (SD) }\end{array}$ \\
\hline ASD & 117 & $93 / 24$ & 5.22 & 11.99 & $\begin{array}{c}\text { Mean } \\
\text { FIQ (SD) }\end{array}$ \\
TD & 81 & $57 / 24$ & 5.88 & 11.95 & $9.67(5.31)$ \\
\hline
\end{tabular}

ASD, autism spectrum disorder; TD, typically developing; FIQ, full-scale intelligence quotient; SD, standard deviation.

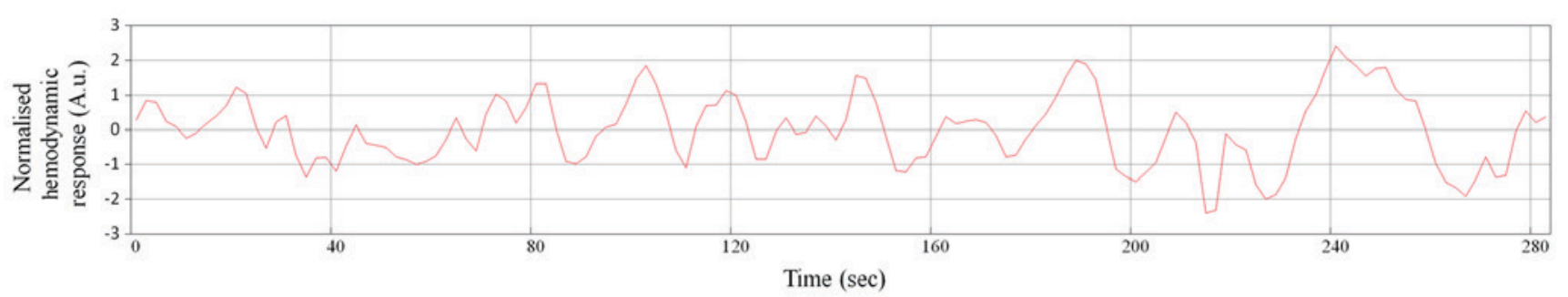

Figure 1. Time series decomposition following IC analysis. Red line represents the time course of IC11. IC, independent component.

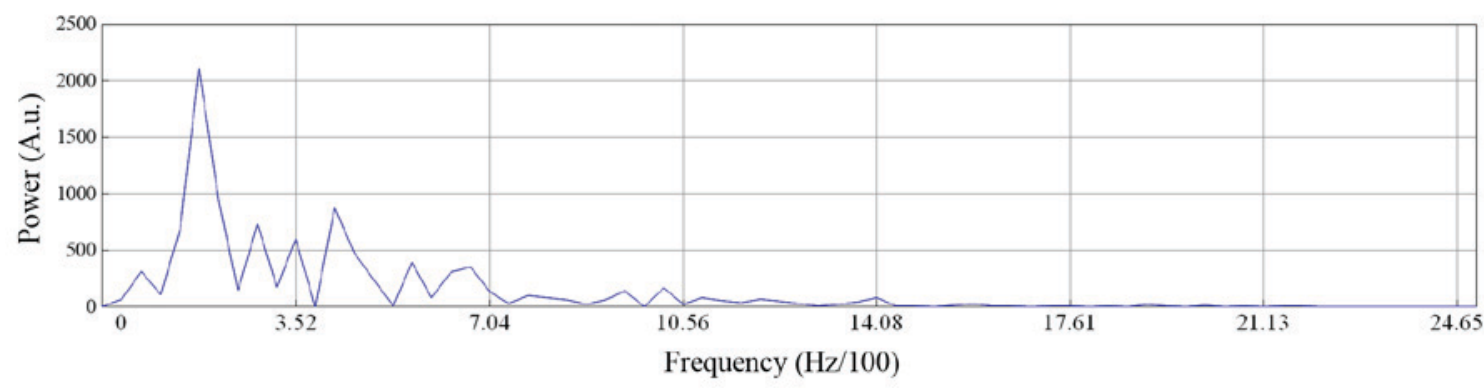

Figure 2. Power spectrum following IC analysis. Blue line represents the power spectrum of IC11. IC, independent component.

were integrated into the proposed computer-aided diagnosis method, as shown in Fig. 4. AE is a key substructure of the SAE, with a symmetrical architecture (Fig. 5). The outputs of each layer in SAE were wired to the inputs of the successive layer. As shown in Fig. 6, the structure of the enhanced SAE proposed in the current study contains 10 layers: An input layer, 8 hidden layers and an output layer. The 8 hidden layers were divided into 4 groups, each with 2 hidden layers. The numbers of nodes in each of the 4 groups were as follows: $1,280,320,80$ and 20 .
The $i$-th activation unit $\mathrm{a}_{i}^{(l)}$ of the $l$-th layer can be represented by the $s_{l-1}$ units of $(l-1)$-th layer through the weight parameter set $w$, a bias term set $b$ and an activation function $f$ as shown below in Equations 1 and 2:

$$
\begin{gathered}
z_{i}^{(l)}=\sum_{j=1}^{n} W_{i j}^{(l-1)} a_{j}^{(l-1)}+b_{i}^{(l-1,} \\
a_{i}^{(l)}=f\left(z_{i}^{(l)}\right)
\end{gathered}
$$




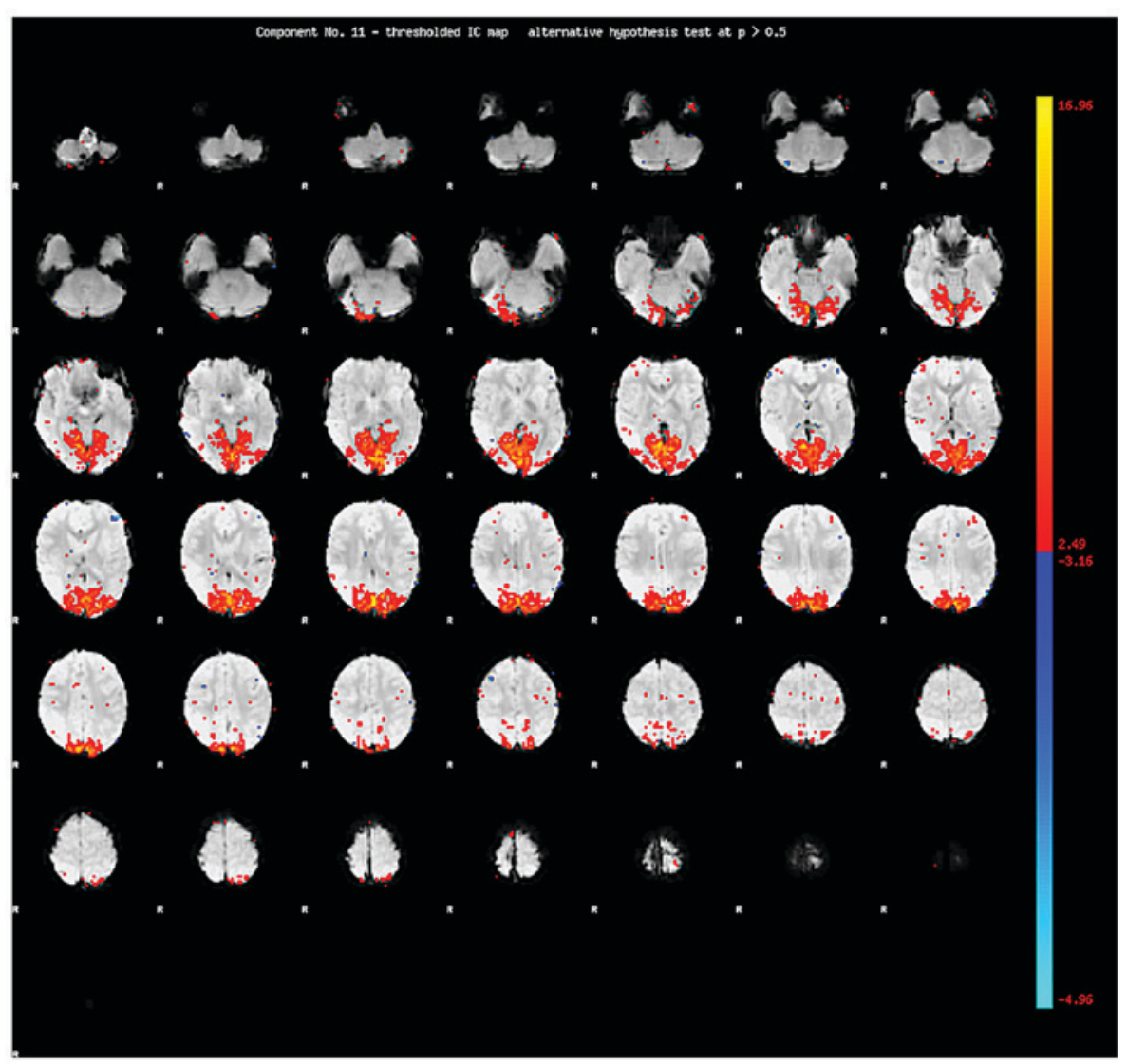

Figure 3. Spatial map of an independent component. Spatial map of IC11. IC, independent component.

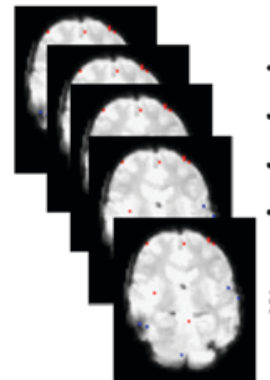

fMRI data

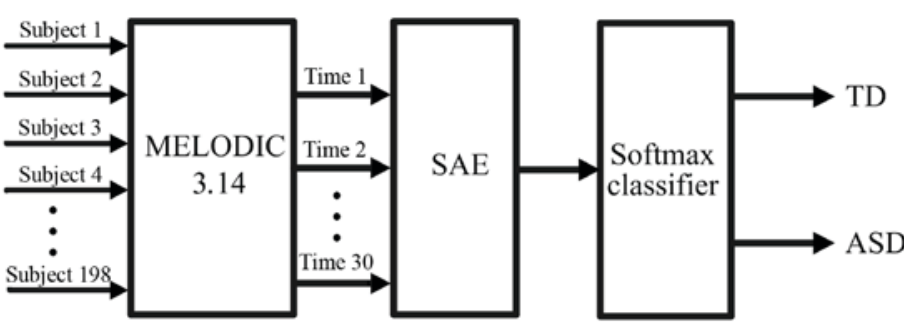

ICA Training the model Predicting type

Figure 4. Proposed classification system. fMRI, functional magnetic resonance imaging; MELODIC, multivariate exploratory linear optimized decomposition into independent components; ICA, independent component analysis; SEA, stacked auto-encoders; TD, typically developing; ASD, autism spectrum disorder.

[ $n=$ number of units in the $(l-1)$-th layer, $f(\bullet)$ was chosen as the sigmoid function].

Given a training dataset with $m$ samples, $\left\{\left(x^{(1)}, y^{(1)}\right), \ldots,\left(x^{(m)}, y^{(m)}\right)\right\}$ where $y^{(i)}$ was the category label of $i$-th sample, $y^{(i)} \in\{1,2, \ldots, k\}$, for the first layer, the activation units were the input data (i.e. $a_{i}^{(1)}=x_{i}$ ). AE enabled the output values to gradually approach the input values by applying a back propagation algorithm. Therefore, the goal of AE was to minimize the distance between the inputs and outputs to learn $w$ and $b$. So the cost function was computed as shown below in Equation 3:

$$
J(W, b)=\left[\frac{1}{m} \sum_{i=1}^{m}\left(\frac{1}{2}\left\|h_{W, b}\left(x^{(i)}\right)-y^{(i)}\right\|^{2}\right)\right]+\frac{\lambda}{2} \sum_{i=1}^{n_{j}-1} \sum_{i=1}^{s_{j}} \sum_{j=1}^{s_{j}-1}\left(W_{j i}^{(i)}\right)^{2}
$$

[ $m$ was the number of training samples, $n_{1}\left(n_{1}=3\right)$ was the number of layers, $s_{l}$ was the number of units in the $l$-th layer and $\lambda$ was the weight decay parameter]. In equation 3 , the first term was the mean square error between input and output values, representing the quality of learning, while the second term was a regularization term that tends to decrease the magnitude of the weights and to prevent the learning from overfitting.

To ensure that the outputs of the hidden layer are desirably sparse, a sparsity constraint must be introduced into the cost function to control the learning process. So the average activation of the $j$-th hidden unit was defined as (with respect to input $x$ ) as shown below in Equation 4:

$$
\hat{\rho}_{j}=\frac{1}{m} \sum_{i=1}^{m}\left(a_{j}^{(2)}\left(x_{i}\right)\right)
$$




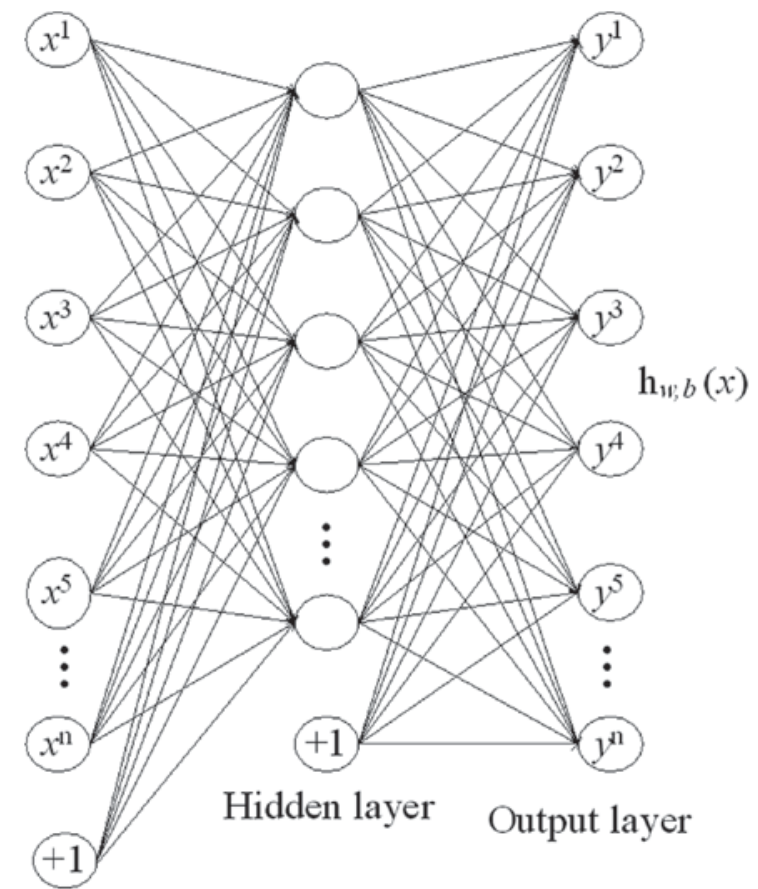

Input layer

Figure 5. Structure of a sparse auto-encoder.

If $\hat{\varrho}_{j}$ was small (close to 0.01 ), most of the units of the hidden layer should be inactive. To force $\hat{\varrho}_{j}$ to be equal to a very small value $\varrho$, the sparsity penalty term was designed based on the concept of Kullback-Leibler divergence, as shown below in Equation 5:

$$
K L\left(\rho \| \hat{\rho}_{j}\right)=\rho \log \frac{\rho}{\hat{\rho}_{j}}+(1-\rho) \log \frac{1-\rho}{1-\hat{\rho}_{j}}
$$

Subsequently, the sparsity penalty term was incorporated into the cost function, as shown below in Equation 6:

$$
J_{\text {słarse }}(W, b)=J(W, b)+\beta \sum K L\left(\rho \| \hat{\rho}_{j}\right)
$$

( $\beta$ was the weight of sparsity penalty term). During the process of learning, the cost function $J_{\text {sparse }}(W, b)$ was minimized by updating $w$ and $b$. As presented in (31), limited-memory BFGS (L-BFGS) was a suitable optimization algorithm for updating $w$ and $b$ in the process of back propagation. In the current study, L-BFGS was adopted, and the softmax regression model was used to classify school-aged children with ASD and TD. After data preprocessing, the time serial matrix was input into the multi-layer SAE, and the output values from the SAE were input into the softmax classifier. The current study used MELODIC (version 3.14), an analysis tool used to decompose each participant's fMRI data into ICs. The time-series was extracted from each IC, and used to generate a vector time-series of each participant with 30 time-series. Similarly, the system was used to obtain 198 vector time-series, and all vector time-series were normalized to the same dimension in order to build a matrix. The matrix was divided into training matrix and test matrix according to the different fold cross-validation (CV). The training matrix was input into the SAE for optimization training.
Finally, the trained multi-layer SAE and softmax classifier were used to classify participants of the testing matrix. In this cycle, multiple test results can be obtained. In the current study, the classification accuracy of all subjects was computed using a CV. For example, in the 198 -fold CV, the original samples are randomly partitioned into 198 groups, with each group including one participant. Of the 198 groups, 197 groups (197 participants) were chosen as the training set, and the remaining one participant was used as the test data. The validation was repeated 198 times such that each participant was used exactly once as the test data. Averaging the 198 results during the validation produced the final estimation. The classification statistical indicators included true positive (TP), false negative (FN), true negative $(\mathrm{TN})$ and false positive (FP) values. The parameters of accuracy, sensitivity, specificity, positive prediction value (PPV) and negative prediction value (NPV) were computed using the following formulae: Accuracy $=(\mathrm{TP}+\mathrm{TN}) /(\mathrm{TP}+\mathrm{TN}+\mathrm{FP}+\mathrm{FN})$ $\mathrm{x} 100 \%$ (7); sensitivit $\mathrm{y}=\mathrm{TP} /(\mathrm{TP}+\mathrm{FN}) \quad \mathrm{x} 100 \%$ (8); specificity $=\mathrm{TN} /(\mathrm{TN}+\mathrm{FP}) \times 100 \%(9) ; \mathrm{PPV}=\mathrm{TP} /(\mathrm{TP}+\mathrm{FP})$ $\mathrm{x} 100 \%$ (10); and NPV=TN/(TN+FN) x100\% (11).

The sensitivity measured the proportion of positive results that were correctly identified. Specificity measured the proportion of negative results that were correctly identified, or the percentage of TD children who were correctly identified as TD. The whole process was performed using Dell Precision T5810 (CPU: Xeon (R), 3.5 GHz; MM: 128 GB; OS: 64-bit Windows 10 professional edition) and Matlab R2012a (7.14.0.739, 64 bits) was used to run the multi-layer SAE and softmax regression algorithm. The specific algorithm settings including, the weight decay, sparsity penalty term, weight of sparsity term and the number of nodes, are summarized in Table III.

\section{Results}

In the current study, the classification accuracy was evaluated using $\mathrm{CV}$. The classifier assigned a label $(\mathrm{ASD}=1, \mathrm{TD}=2)$ to each participant, and the total accuracy was computed for each category. The accuracy, specificity, sensitivity, PPV and NPV for each $\mathrm{CV}$ are summarized in Table IV.

With the same 198 subject data and 198 -fold CV, the SAE, probabilistic neural network (PNN) and support vector machine (SVM) algorithms achieved 96.97, 84.58 and $83.69 \%$ accuracy, respectively (Table V). Therefore, the proposed SAE with 8 hidden layers of the current study was more accurate than other machine learning algorithms. These results demonstrated that the SAE algorithm was a more effective method for the analysis and processing of data in the current study. In addition, the 66 -fold CV results revealed that the proposed SAE algorithm produced a better balance between the classification accuracy and computation time compared with SAE algorithms with different numbers of hidden layers (data not shown). The average accuracy obtained using the SAE algorithm with 6,8 and 10 hidden layers was $93.69,98.99$ and $95.90 \%$, respectively (data not shown). However, the computation time taken for SAE algorithms with 6,8 and 10 numbers of hidden layers was 70,018, 68,588 and 75,891 sec, respectively (data not shown). In conclusion, increasing the number of hidden layers did not improve the accuracy of the SAE algorithm, however the running time 
Table III. Algorithm parameter values used in the 8 hidden layers.

\begin{tabular}{lccrr}
\hline Hidden layer & Weight decay & Sparsity penalty term & Weight of sparsity penalty term & Number of node \\
\hline No. 1,2 & $1 \times 10^{-8}$ & 0.01 & 3 & 1,280 \\
No. 3,4 & $1 \times 10^{-8}$ & 0.01 & 3 & 320 \\
No. 5,6 & $1 \times 10^{-8}$ & 0.01 & 3 & 80 \\
No. 7,8 & $1 \times 10^{-8}$ & 0.01 & 30 \\
\hline
\end{tabular}

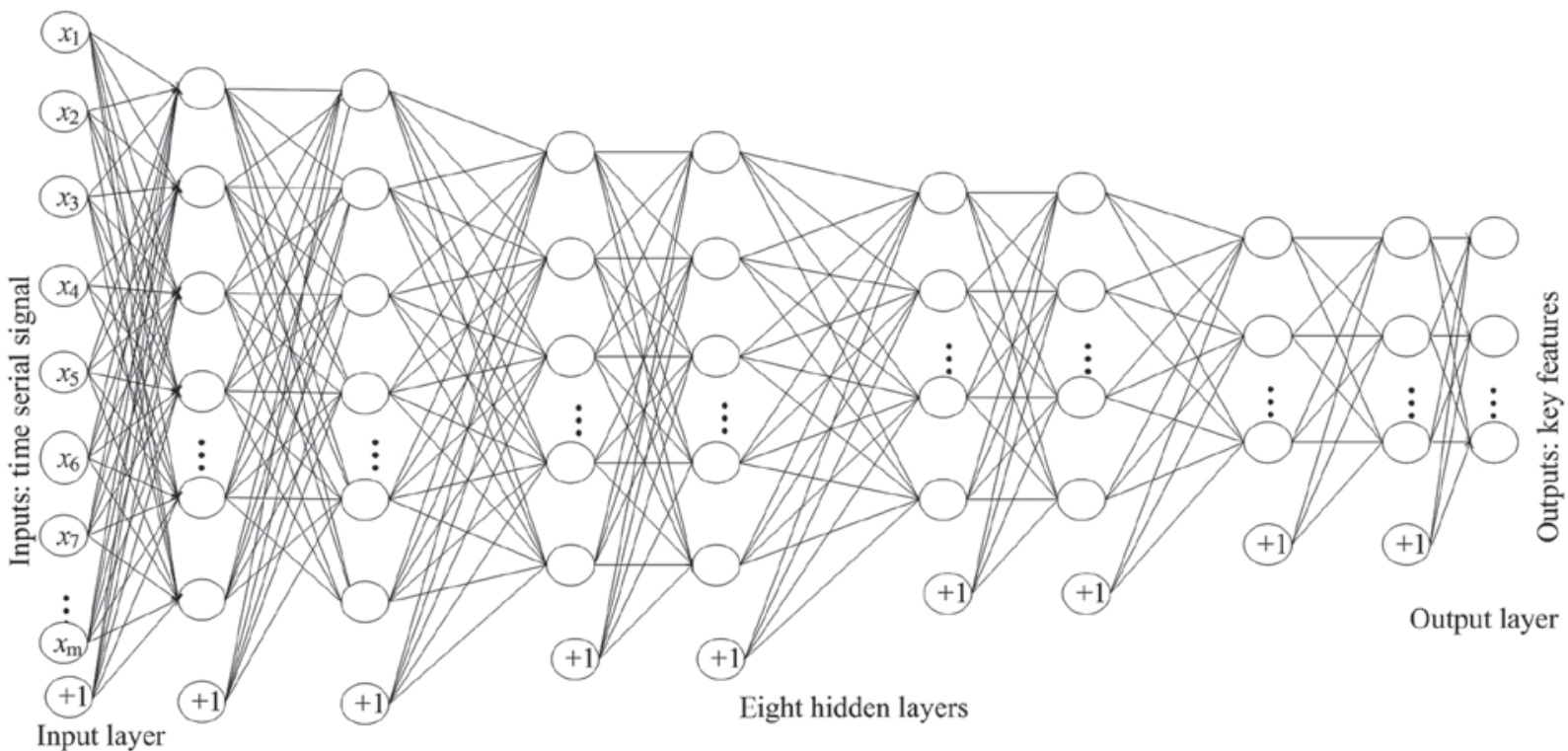

Figure 6. Schematic architecture of an enhanced stacked auto-encoder.

increased. These results suggest that the SAE algorithm with 8 hidden layers can balance the classification accuracy and running time.

The current study used a novel deep learning method with full brain frequency, to generate a higher diagnostic accuracy $(96.26 \%)$, with increased sensitivity $(98.03 \%)$ and specificity (93.62\%). Furthermore, the accuracy classification analysis of the current study was compared with previously published studies (Table VI). The results demonstrated that the classification accuracy was $\sim 15 \%$ higher in the current study compared with previous studies, where only one or two frequency bands were used (19,33,38-40).

\section{Discussion}

In the current study, the proposed SAE algorithm-based classification system was generated using all frequency bands using multi-institutional fMRI datasets to produce high classification accuracy to distinguish school-aged children (5-12 years) with ASD from those TD. The present study examined an auxiliary method to distinguish ASD with high sensitivity and specificity, which was considered as an entirely hypothesis-free and data-driven method. In a previous study, ASD (12-42 years) and TD (8-39 years) participants were classified with relatively low accuracy (38). As ASD is an early onset disorder with variable developmental trajectory, Uddin et al (33) used a network-based classification method to predict children (7-13 years) with ASD and those TD. Although Uddin et al (33) examined 10 whole-brain functional networks (salience, central executive, posterior default mode, ventral default mode, anterior default mode, dorsal attention, motor, visual association, primary visual and frontotemporal), the salience network was the only network, which demonstrated good classification accuracy. In the current study, several other brain networks were integrated, and the classification accuracy was increased compared with single brain network classification as demonstrated by Uddin et al (33). Another study classifying ASD and TD participants through a default network only achieved a relatively low accuracy compared with the current study (39). Although previous studies gained some success in mechanism research, previous studies used relatively low numbers of participants and the results were based on single brain networks only. However, the data-driven method used in the current study used a bigger dataset, and attained a higher accuracy by not only integrating single brain networks, but also measuring the joint effect of several brain networks. In 2015, Iidaka (40) used a PNN approach on 640 participants to distinguish between ASD and TD, with relatively high accuracy.

Following a comparison with previously published studies using PNN and SVM methods, the current study revealed that the SAE algorithm achieved a higher classification accuracy compared with traditional machine learning algorithm. In 
Table IV. Cross-validation classification accuracy analysis.

\begin{tabular}{lcccccc}
\hline Parameter & 11-fold $(\%)$ & 33-fold $(\%)$ & 66-fold $(\%)$ & 99-fold (\%) & 198-fold (\%) & Average, $\%(\mathrm{SD})$ \\
\hline Accuracy & 94.19 & 93.69 & $98.99^{\mathrm{a}}$ & 97.47 & 96.97 & $96.26(2.02)$ \\
Specificity & 87.04 & 87.23 & $100^{\mathrm{a}}$ & 96.30 & 97.53 & $93.62(5.43)$ \\
Sensitivity & $99.15^{\mathrm{a}}$ & 97.86 & 98.29 & 98.29 & 96.58 & $98.03(0.84)$ \\
PPV & 91.70 & 91.97 & $100^{\mathrm{a}}$ & 97.46 & 98.26 & $95.88(3.40)$ \\
NPV & $98.60^{\mathrm{a}}$ & 96.60 & 97.59 & 97.50 & 95.18 & $97.09(1.15)$ \\
\hline
\end{tabular}

${ }^{a}$ Maximum value for each parameter analyzed. PPV, positive prediction value; NPV, negative prediction value; SD, standard deviation.

Table V. Comparison of classification accuracy of three different algorithms.

\begin{tabular}{lccr}
\hline & Stacked auto-encoders (\%) & Probabilistic neural network (\%) & Support vector machine (\%) \\
\hline Accuracy & 96.97 & 84.58 & 83.69 \\
\hline
\end{tabular}

Table VI. Comparison between the classification accuracy analysis obtained in the current study and previously published studies.

\begin{tabular}{|c|c|c|c|c|c|c|c|}
\hline Author, year & $\begin{array}{c}\text { Functional } \\
\text { networks }\end{array}$ & $\begin{array}{c}\text { Accuracy } \\
(\%)\end{array}$ & $\begin{array}{c}\text { Sensitivity } \\
(\%)\end{array}$ & $\begin{array}{c}\text { Specificity } \\
(\%)\end{array}$ & $\begin{array}{l}\text { PPV } \\
(\%)\end{array}$ & $\begin{array}{l}\text { NPV } \\
(\%)\end{array}$ & (Refs.) \\
\hline Current study & & 96.26 & 98.03 & 93.62 & 95.88 & 97.09 & - \\
\hline \multirow[t]{10}{*}{ Uddin et al, 2013} & Salience & 78.00 & 75.00 & 80.00 & 79.00 & 76.00 & (33) \\
\hline & Central executive & 58.00 & 55.00 & 60.00 & 58.00 & 57.00 & (33) \\
\hline & Posterior default mode & 63.00 & 65.00 & 60.00 & 62.00 & 63.00 & (33) \\
\hline & Ventral default mode & 60.00 & 55.00 & 65.00 & 61.00 & 59.00 & (33) \\
\hline & Anterior default mode & 63.00 & 50.00 & 75.00 & 67.00 & 60.00 & (33) \\
\hline & Motor & 68.00 & 60.00 & 75.00 & 71.00 & 65.00 & (33) \\
\hline & Visual association & 65.00 & 65.00 & 65.00 & 65.00 & 65.00 & (33) \\
\hline & Dorsal attention & 73.00 & 75.00 & 70.00 & 71.00 & 74.00 & (33) \\
\hline & Primary visual & 73.00 & 60.00 & 85.00 & 80.00 & 68.00 & $(33)$ \\
\hline & Frontotemporal & 68.00 & 60.00 & 75.00 & 71.00 & 65.00 & (33) \\
\hline Anderson et al, 2011 (subject $<20$ years) & & 89.00 & 83.00 & 95.00 & - & - & $(38)$ \\
\hline Chen et al, 2016 (NYU dataset) & & 80.00 & 95.00 & 72.00 & - & - & $(19)$ \\
\hline Chen et al, 2016 (UM dataset) & & 84.00 & 69.00 & 94.00 & - & - & (19) \\
\hline Chen et al, 2016 (USM dataset) & & 80.00 & 80.00 & 80.00 & - & - & (19) \\
\hline Iidaka, 2015 (50-fold CV) & & 90.30 & 92.30 & 88.40 & 88.30 & 92.40 & $(40)$ \\
\hline Iidaka, 2015 (10-fold CV) & & 86.90 & 85.90 & 87.80 & 87.00 & 86.70 & $(40)$ \\
\hline Iidaka, 2015 (2-fold CV) & & 77.20 & 75.00 & 79.30 & 77.50 & 76.90 & $(40)$ \\
\hline
\end{tabular}

PPV, positive prediction value; NPV, negative prediction value; Refs., references; NYU, New York University; UM, University of Michigan; USM, Universiti Sains Malaysia; CV, cross-validation.

2016, Chen et al (19) used SVM to distinguish between ASD and TD, and obtained relatively low classification accuracy. Although Chen et al (19) and Iidaka (40) used larger datasets, which were obtained from the same database, the 2 classification algorithms (SVM and PNN) demonstrated less accuracy compared with the current study. The differences in methodology between the SAE and SVM/PNN algorithms may account for the differences observed in classification accuracy of the above-mentioned studies and the current study.

In conclusion, a deep learning based approach was used to successfully distinguish 198 school-aged children with ASD from those TD, using a data-driven method with high accuracy of $96.30 \%$. Taken together, these results demonstrate the potential clinical application of ASD diagnostic tools in school-aged children with ASD. 


\section{Acknowledgements}

The authors would like to thank the researchers and funding agencies that contributed to the ABIDE II database.

\section{Funding}

The current study was funded by grants from The Natural Science Foundation of China (grant nos. 61662047 and 61463035).

\section{Availability of data and materials}

All datasets used and/or analyzed during the current study are available from the corresponding author in reasonable request. In addition, the original functional magnetic resonance imaging datasets can be obtained from the Autism Brain Imaging Data Exchange II database (fcon_1000.projects.nitrc. org/indi/abide/index.html).

\section{Authors' contributions}

ZX designed the experiment, analyzed the functional magnetic resonance imaging (fMRI) data, interpreted the results and prepared the manuscript. JW and CW contributed significantly to the conception and design of the work, and were involved in the collection and interpretation of data. XY prepared all the figures in the manuscript and detected the stacked auto-encoders algorithm. NJ performed the preprocessing of the fMRI data. All authors read and approved the final manuscript.

\section{Ethical approval and consent to participate}

The current study was approved by the Ethics Committee of Nanchang University. In addition, the ABIDE II database allows unrestricted use for non-commercial purposes.

\section{Patient consent for publication}

Not applicable.

\section{Competing interests}

The authors declare that they have no competing interests.

\section{References}

1. Belmonte MK, Allen G, Beckel-Mitchener A, Boulanger LM, Carper RA and Webb SJ: Autism and abnormal development of brain connectivity. J Neurosci 24: 9228-9231, 2004.

2. Developmental Disabilities Monitoring Network Surveillance Year 2010 Principal Investigators; Centers for Disease Control and Prevention (CDC): Prevalence of autism spectrum disorder among children aged 8 years-autism and developmental disabilities monitoring network, 11 sites, United States, 2010. MMWR Surveill Summ 63: 1-22, 2014.

3. McCarthy M: Autism diagnoses in the US rise by $30 \%$, CDC reports. BMJ 348: g2520, 2014.

4. Experts: Large Numbers of children under 3 with autism go undiagnosed. http://china.caixin.com/2018-10-25/101338630. html. Accessed on October 25, 2018.

5. Xiao Z, Wang C, Nan J and Wu J: SAE-based classification of school-aged children with autism spectrum disorders using functional magnetic resonance imaging. Multimed Tools Applicat 77: 22809-22820, 2018.
6. Holzer L, Mihailescu R, Rodrigues-Degaeff C, Junier L, Muller-Nix C, Halfon $\mathrm{O}$ and Ansermet F: Community introduction of practice parameters for autistic spectrum disorders: Advancing early recognition. J Autism Dev Disord 36: 249-262, 2006.

7. Goh S, Dong Z, Zhang Y, DiMauro S and Peterson BS: Mitochondrial dysfunction as a neurobiological subtype of autism spectrum disorder: Evidence from brain imaging. JAMA Psychiatry 71: 665-671, 2014.

8. Lord C, Rutter M and Le Couteur A: Autism Diagnostic Interview-Revised: A revised version of a diagnostic interview for caregivers of individuals with possible pervasive developmental disorders. J Autism Dev Disord 24: 659-685, 1994.

9. Bastiaansen JA, Meffert H, Huizinga P, Ketelaars C, Pijnenborg M, Bartels A, Minderaa R, Keysers C and de Bildt A: Diagnosing Autism Spectrum Disorders in Adults: the Use of Autism Diagnostic Observation Schedule (ADOS) Module 4. J Autism Dev Disord 41 (9): 1256-1266, 2011.

10. Sungur MZ and Gündüz A: A comparison of DSM-IV-TR and DSM-5 definitions for sexual dysfunctions: Critiques and challenges. J Sex Med 11: 364-373, 2014.

11. Dosreis S, Weiner CL, Johnson L and Newschaffer CJ: Autism spectrum disorder screening and management practices among general pediatric providers. J Dev Behav Pediatr 27: S88-S94, 2006.

12. Matson JL and Goldin RL: Diagnosing young children with autism. Int J Dev Neurosci 39: 44-48, 2014.

13. Di Martino A, Ghaffari M, Curchack J, Reiss P, Hyde C, Vannucci M, Petkova E, Klein DF and Castellanos FX: Decomposing intra-subject variability in children with attention-deficit/hyperactivity disorder. Biol Psychiatry 64: 607-614, 2008.

14. Hoptman MJ, Zuo XN, Butler PD, Javitt DC, D'Angelo D, Mauro CJ and Milham MP: Amplitude of low-frequency oscillations in schizophrenia: A resting state fMRI study. Schizophr Res 117: 13-20, 2010.

15. Han Y, Wang J, Zhao Z, Min B, Lu J, Li K, He Y and Jia J: Frequency-dependent changes in the amplitude of low-frequency fluctuations in amnestic mild cognitive impairment: A resting-state fMRI study. Neuroimage 55: 287-295, 2011.

16. Barttfeld P, Wicker B, Cukier S, Navarta S, Lew S, Leiguarda R and Sigman M: State-dependent changes of connectivity patterns and functional brain network topology in autism spectrum disorder. Neuropsychologia 50: 3653-3662, 2012.

17. Bajaj S, Adhikari BM and Dhamala M: Higher frequency network activity flow predicts lower frequency node activity in intrinsic low-frequency BOLD fluctuations. PLoS One 8: e64466, 2013.

18. Deshpande G, Libero LE, Sreenivasan KR, Deshpande HD and Kana RK: Identification of neural connectivity signatures of autism using machine learning. Front Hum Neurosci 7: 670, 2013.

19. Chen H, Duan X, Liu F, Lu F, Ma X, Zhang Y, Uddin LQ and Chen H: Multivariate classification of autism spectrum disorder using frequency-specific resting-state functional connectivity-A multi-center study. Prog Neuropsychopharmacol Biol Psychiatry 64: 1-9, 2016.

20. Zuo XN, Di Martino A, Kelly C, Shehzad ZE, Gee DG, Klein DF, Castellanos FX, Biswal BB and Milham MP: The oscillating brain: Complex and reliable. Neuroimage 49: 1432-1445, 2010.

21. Penttonen M and Buzsáki G: Natural logarithmic relationship between brain oscillators. Thalamus Relat Syst 2: 145-152, 2003.

22. Buzsáki G and Draguhn A: Neuronal oscillations in cortical networks. Science 304: 1926-1929, 2004.

23. Siegel M, Donner TH and Engel AK: Spectral fingerprints of large-scale neuronal interactions. Nat Rev Neurosci 13: 121-134, 2012.

24. Lynch CJ, Uddin LQ, Supekar K, Khouzam A, Phillips J and Menon V: Default mode network in childhood autism: Posteromedial cortex heterogeneity and relationship with social deficits. Biol Psychiatry 74: 212-219, 2013.

25. Balsters JH, Mantini D and Wenderoth N: Connectivity-based parcellation reveals distinct cortico-striatal connectivity fingerprints in Autism Spectrum Disorder. Neuroimage 170: 412-423, 2018.

26. Risk BB, Matteson DS, Ruppert D, Eloyan A and Caffo BS: An evaluation of independent component analyses with an application to resting-state fMRI. Biometrics 70: 224-236, 2014

27. Shin HC, Orton MR, Collins DJ, Doran SJ and Leach MO: Stacked autoencoders for unsupervised feature learning and multiple organ detection in a pilot study using 4D patient data. IEEE Trans Pattern Anal Mach Intell 35: 1930-1943, 2013. 
28. Autism Brain Imaging Data Exchange II, 2016. http://fcon_1000. projects.nitrc.org/indi/abide/abide_II.html. Accessed Aug 30, 2012.

29. Assaf M, Jagannathan K, Calhoun VD, Miller L, Stevens MC Sahl R, O'Boyle JG, Schultz RT and Pearlson GD: Abnormal functional connectivity of default mode sub-networks in autism spectrum disorder patients. Neuroimage 53: 247-256, 2010.

30. Di Martino A, Kelly C, Grzadzinski R, Zuo XN, Mennes M, Mairena MA, Lord C, Castellanos FX and Milham MP: Aberrant striatal functional connectivity in children with autism. Biol Psychiatry 69: 847-856, 2011.

31. Perez Velazquez JL, Barcelo F, Hung Y, Leshchenko Y, Nenadovic V, Belkas J, Raghavan V, Brian J and Garcia Dominguez L: Decreased brain coordinated activity in autism spectrum disorders during executive tasks: Reduced long-range synchronization in the fronto-parietal networks. Int J Psychophysiol 73: 341-349, 2009.

32. Fagiolo $\mathrm{G}$, Waldman A and Hajnal JV: A simple procedure to improve FMRIB Software Library Brain Extraction Tool performance. Br J Radiol 81 (963): 250, 2008

33. Uddin LQ, Supekar K, Lynch CJ, Khouzam A, Phillips J, Feinstein C, Ryali S and Menon V: Salience network-based classification and prediction of symptom severity in children with autism. JAMA Psychiatry 70: 869-879, 2013.

34. Li YO, Adali T and Calhoun VD: Estimating the number of independent components for functional magnetic resonance imaging data. Hum Brain Mapp 28: 1251-1266, 2007.

35. Beckmann CF, Noble JA and Smith SM: Investigating the intrinsic dimensionality of FMRI data for ICA. Neuroimage 13: S76, 2001.
36. McKeown MJ and Sejnowski TJ: Independent component analysis of fMRI data: Examining the assumptions. Hum Brain Mapp 6: 368-372, 1998

37. Jiang M, Liang Y, Feng X, Fan X, Pei Z, Xue Y and Guan R: Text classification based on deep belief network and softmax regression. Neural Comput Applicat 29: 61-70, 2018.

38. Anderson JS, Nielsen JA, Froehlich AL, DuBray MB, Druzgal TJ, Cariello AN, Cooperrider JR, Zielinski BA, Ravichandran C, Fletcher PT, et al: Functional connectivity magnetic resonance imaging classification of autism. Brain 134: 3742-3754, 2011.

39. Murdaugh DL, Shinkareva SV, Deshpande HR, Wang J, Pennick MR and Kana RK: Differential deactivation during mentalizing and classification of autism based on default mode network connectivity. PLoS One 7: e50064, 2012.

40. Iidaka T: Resting state functional magnetic resonance imaging and neural network classified autism and control. Cortex 63: $55-67,2015$. 